\title{
Optimism surrounds new targeted therapies for pancreatic neuroendocrine tumors
}

U ntil now, the treatment options available for patients with pancreatic neuroendocrine tumors (NETs) have been limited. That situation may be about to change with the findings of two phase III, multicenter, double-blind randomized, placebo-controlled trials published in the New England Journal of Medicine.

Eric Raymond and colleagues studied sunitinib malate - a tyrosine kinase inhibitor-because of its success in phase I and II trials in patients with NETs (treatment response and sustained tumor stabilization) and increased understanding of its mechanism of action in pancreatic NETs. Their primary hypothesis was that sunitinib could improve an expected median progression-free survival (PFS) of 5 months by $50 \%$.

To ensure the trial was adequately powered, initial plans were to recruit 340 patients with advanced and/or metastatic pancreatic NETs and documented disease progression in the previous 12 months. Patients were randomly allocated to receive sunitinib $37.5 \mathrm{mg}$ per day or placebo-all received best supportive care. During the trial, patients in the placebo arm whose disease progressed had sunitinib made available to them via two continuation trials.

This trial was terminated early, after just 171 patients had been recruited, as sunitinib more than doubled PFS (from 5.5 months to 11.4 months) - patients on placebo at that time were switched to sunitinib. Sunitinib also provided benefit in terms of overall survival (hazard ratio for death 0.41), tumor response and sustained tumor stabilization, regardless of the extent of the disease, its proliferation index or the use of somatostatin analogs. "The speed of activity was also impressive for some patients who had symptom relief as early as 3-4 days after treatment initiation," explains Raymond.

Raymond and colleagues now plan to study the effect of sunitinib on several subtypes of NET that have unmet needs: poorly differentiated NETs (trial ongoing), carcinoids (trial planned) and other nongastrointestinal endocrine tumors. "We would also like to know whether we can use sunitinib in a curative intent prior to surgery or in an adjuvant setting after surgery," says Raymond.

In the RADIANT-3 trial, James Yao and colleagues studied the effect of everolimus, a mammalian target of rapamycin (mTOR) inhibitor. "Several genetic cancer syndromes in the mTOR pathway are associated with NETs, and dysregulation of this pathway in sporadic tumors is linked to poor prognosis," explains Yao. The researchers were also encouraged by promising results in phase I and II trials in patients with NETs.

\section{We now have two efficient \\ treatments for pancreatic NETs, where last year we had none}

To test the hypothesis that everolimus would delay tumor growth in patients with pancreatic NETs, Yao et al. randomly allocated patients who had experienced radiologic progression of their tumors (advanced, intermediate grade or lowgrade) in the previous 12 months to everolimus $10 \mathrm{mg}$ per day $(n=207)$ or placebo $(n=203)$. All received best supportive care. Patients with radiologic progression during the study were unblinded; those on placebo were given the option to take open-label everolimus.

"Everolimus therapy led to a statistically significant and clinically meaningful delay in tumor progression," says Yao. Indeed, the median PFS rose from 4.6 months to 11 months-an increase of more than 6 months-and the risk of tumor progression was reduced by $65 \%$. An estimated $34 \%$ of patients who received everolimus were progression-free at 18 months compared with just $9 \%$ of patients who received placebo.
Limited treatment options and the size of the treatment effect achieved with everolimus lead Yao to be optimistic about its clinical application. "Hopefully, these findings will ultimately lead to a definitive change in the standard of care for those patients with pancreatic NETs."

Yao and colleagues now intend to assess patients who have been treated with everolimus for the presence of somatic mutations in the mTOR pathway, which have recently been identified in unselected patients with pancreatic NETs - they hope this will allow them to identify predictive biomarkers. Other work includes a randomized phase II study of everolimus combined with bevacizumab, an inhibitor of vascular endothelial growth factor. In addition, as resistance to $\mathrm{mTOR}$ inhibitors is thought to involve the insulin growth factor pathway, Yao's team have a phase I study underway to investigate combined treatment with everolimus plus an inhibitor of insulin growth factor receptor.

Importantly, although both sunitinib and everolimus were associated with significantly more adverse events than placebo, most were not severe and could be managed by reducing the drug dose and/or interrupting treatment. The most frequent adverse event associated with sunitinib was diarrhea, whereas with everolimus it was stomatitis.

"We now have two efficient treatments for pancreatic NETs, where last year we had none," concludes Raymond. "I guess if we can draw a parallel with kidney cancer, we now have two drugs that could be used sequentially to delay progression, symptoms and improve survival for the greater benefit of patients."

Natalie J. Wood 